Int. J. Dev. Biol. 51: 265-272 (2007)

doi: $10.1387 / \mathrm{ijdb} .072270 \mathrm{kt}$

Original Article

\title{
Heart formation and left-right asymmetry in separated right and left embryos of a newt
}

\author{
KAZUHIRO TAKANO ${ }^{1}$, YUZURU ITO ${ }^{2}$, SHUICHI OBATA ${ }^{3}$, TSUTOMU OINUMA ${ }^{4}$, SHINJI KOMAZAKI ${ }^{1}$, \\ HIROAKI NAKAMURA ${ }^{1}$ and MAKOTO ASASHIMA ${ }^{2,5,6, *}$
}

\begin{abstract}
${ }^{1}$ Department of Anatomy, Faculty of Medicine, Saitama Medical University, Iruma, Saitama, ${ }^{2}$ International Cooperative Research Program (ICORP), Japan Science and Technology Agency (JST), Department of Life Science (Biology), Graduate School of Arts and Sciences, The University of Tokyo, Tokyo, ${ }^{3}$ Division of Biology, College of Liberal Arts and Sciences, Kitasato University, Sagamihara, Kanagawa, ${ }^{4}$ Department of Anatomy Ultrastructural Cell Biology, Faculty of Medicine, University of Miyazaki, Kiyotake, Miyazaki, ${ }^{5}$ National Institute of Advanced Industrial Sciences and Technology (AIST), Tsukuba, Ibaraki and ${ }^{6}$ Department of Life Science (Biology), Graduate School of Arts and Sciences, The University of Tokyo, Meguro-ku, Tokyo, Japan.
\end{abstract}

\begin{abstract}
During vertebrate cardiac development, the heart tube formed by fusion of right and left presumptive cardiac mesoderms (PCMs) undergoes looping toward the right, resulting in an asymmetrical heart. Here, we examined the right and left PCMs with regard to heart-tube looping using right- and left-half newt embryos (Cynops pyrrhogaster). In the half embryos, the rightward (normal) loop of the heart tube was formed from the left PCM, irrespective of the timing of its separation, while the leftward (reversed) loop of the heart tube was formed from the right PCM, separated by stage 18. In addition, the direction of the leftward loop was inverted to the rightward direction in right-half embryos bisected after stage 18. Incision or resection of the embryonic caudal region implicated interactions between the right and left sides of this region as crucial for inverting the direction of the heart-tube loop from leftward to rightward in the right-half embryos. In situ hybridization of $C y$ Nodal (Cynops nodal-related gene) suggested that the inversion of heart looping in the right-half embryos has no association with the CyNodal expression pattern. Based on these findings, we propose a mechanism for the rightward looping underlying normal amphibian cardiac development.
\end{abstract}

KEY WORDS: Cynops pyrrhogaster, cardiac development, heart rudiment, cardiac looping, newt

\section{Introduction}

The external morphology of metazoans is symmetric, but the shape and arrangement of their internal organs are often asymmetric. Asymmetry appears earlier in the heart than in any other organ during embryonic development. The vertebrate heart is formed by fusion of the symmetrically distributed right and left presumptive cardiac mesoderms (PCMs). The right and left PCMs migrate to the thorax of the embryo, where they fuse to form a heart tube. The heart tube then undergoes looping toward the right, resulting in the formation of atria and ventricles (reviewed by Manner, 2000).

Mechanisms by which this heart tube directionality is determined have been extensively studied. Most of these studies investigated the direction of heart-tube loops formed after fusion of the right and left PCMs (reviewed by Levin, 2005; Ramsdell,
2005). In contrast, Spemann and Falkenberg (1919) demonstrated that when a two-cell newt embryo was ligated along the first cleavage plane, two complete embryos developed from each blastomere. In each of these two separated embryos, a heart was formed, with the heart-tube loops directed rightward (normal direction) in the left-half embryos $(n=2)$ and half of the loops directed leftward (reversed) in the right-half embryos $(n=2)$. Hoyle et al. (1992) demonstrated in chick that when the right and left PCMs were exchanged between donor and host embryos at the neurula stage, double-right-sided embryos (two areas of PCM of the same right sidedness) formed heart-tube loops directed leftward in almost $50 \%$ of cases. These findings suggested that any intrinsic difference in heart-tube loop formation between the

Abbreviations used in this paper: PCM, presumptive cardiac mesoderm.

\footnotetext{
*Address correspondence to: Makoto Asashima. Department of Life Science (Biology), Graduate School of Arts and Sciences, The University of Tokyo, 38-1 Komaba, Meguro-ku, Tokyo 153-8902, Japan. Fax: +81-3-5454-4330. e-mail: asashi@bio.c.u-tokyo.ac.jp
} 
A
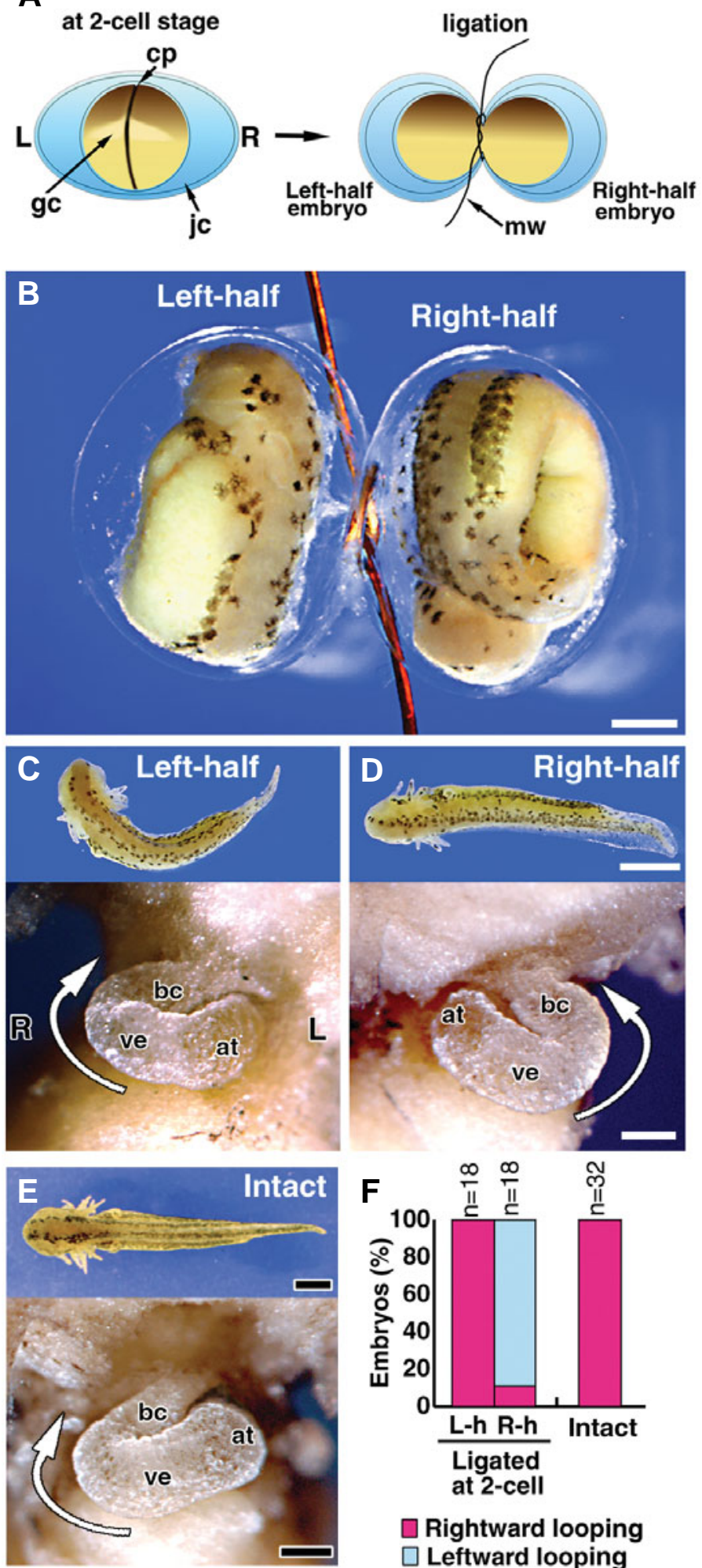

\section{$\square$ Rightward looping $\square$ Leftward looping}

right and left PCMs was derived from the right and left sides of an embryo, raising the question as to how both PCMs cannot fail to form a rightward loop after fusion in spite of their different properties.

The present study therefore sought to address the following questions using separated right- and left-half embryos of newt. Can separated right and left PCM form normal heart tubes, respectively? What direction will be taken by the looping of each heart tube, if it is assumed that each PCM formed an original heart tube? Finally, what influences do the individual right and left PCMs exert on the rightward looping of the heart tube formed after fusion of the PCMs?

Recent studies have also implicated asymmetrically expressed genes in the determination of embryonic left-right asymmetry, including Nodal (Levin et al., 1995; Collignon et al., 1996; Lowe etal., 1996; Lohr etal., 1997; Patel etal., 1999; Mogi etal., 2003; Toyoizumi et al., 2005), Pitx2 (Campione etal., 1999; Patel etal., 1999; Campione et al., 2001; Yu et al., 2001; Mogi et al., 2003; Toyoizumi etal., 2005), BMP (Ramsdell and Yost, 1999; Branford et al., 2000; Monsoro-Burq and Le Douarin, 2000; Monsoro-Burq and Le Douarin, 2001) and SnR (Issac et al., 1997, Patel et al., 1999). Of these genes, Nodal, which is expressed only in the left side of the embryo, is important in determining embryonic leftright asymmetry across many species (Levin etal., 1995; Collignon etal., 1996; Lowe etal., 1996; Lohr etal., 1997; Patel etal., 1999; Mogi et al., 2003; Toyoizumi et al., 2005). Therefore, in the present study, we additionally analyzed the relationship between the heart-tube loop direction in right- and left-half embryos and the expression of CyNodal, a noda/related gene of newt (Ito et al., 2006), therein.

\section{Results}

\section{Looping of the heart tube in ligated embryos}

First, we revisited the twinning experiments of Spemann and Falkenberg (1919) using two-cell stage newt embryos and extended on their findings. Stage 2 (two-cell) embryos were ligated along the cleavage furrow together with the jelly capsule to obtain two blastomeres (Fig. 1A). The symmetrically divided embryos underwent development at the same pace as normal embryos (Fig. 1B). At stage 37, both right- and left-half embryos showed normal and similar external morphology (Fig. 1C, D, upper), although each half embryo was shorter in length than intact normal embryos (Fig. 1E, upper). The rightward loops of the heart tubes were formed in $100 \%$ of left-half embryos (Fig. 1C, lower, F), identical to intact embryos (Fig. 1E, lower). In contrast, the leftward loops of the heart tubes were formed in $89 \%$ of right-half embryos (Fig. 1D, lower, F). All of the hearts formed in the rightand left-half embryos had normal external morphology compared

Fig. 1. Hearts formed in ligated embryos. (A) Schematic representation of the ligating experiment. $c p$, cleavage plane; gc, grey crescent; jc, jelly capsule; mw, metal wire; L, left; $R$, right. (B) Right-and left-half embryos with complete external morphology (stage 37) formed from two blastomeres of an embryo ligated at the two-cell stage. Scale bar: $0.5 \mathrm{~mm}$. (C,D) Rightand left-half embryos shown in (B), viewed from the dorsal side (upper pictures) and the heart tubes formed in each embryo viewed from the ventral side (lower pictures). The embryos were taken out of the jelly capsule and fertilization membrane. Portions of the body wall and pericardium were removed to expose the heart. bc, bulbus cordis; at, atrium; ve, ventricle. The white arrow indicates the direction of the heart-tube loop. Scale bar: $1 \mathrm{~mm}$ (upper pictures), $0.1 \mathrm{~mm}$ (lower pictures). (E) Intact normal embryo at stage 37. Scale bar: $1 \mathrm{~mm}$ (upper picture), $0.1 \mathrm{~mm}$ (lower picture). (F) Graphic representation of the percentage direction taken by each heart-tube loop formed in the ligated embryos. L-h, left-half embryo; R-h, right-half embryo. 
with intact embryos, with the exception of the loop direction. These results indicate that each of the right- and left-half embryos separated at first cleavage could form looping heart tubes, but that the loop direction assumed a mirror image relationship to each other. This series of experiments did not, however, provide information about the developmental potential of right and left PCMs, since these structures were not apparent at the two-cell stage. We therefore adopted another experimental approach to address this issue.

\section{Looping of the heart tube in bisected embryos}

To investigate the ability of right and left PCMs to form the heart-tube loops, we bisected embryos through the center of the blastopore at several stages from the appearance of PCM (stage 11) to immediately before fusion of the left and right PCMs (stage 25) (Fig. 2A, F, lower). The right- and left-half embryos were incubated until stages 35-37 when the loops of the heart tubes became evident. The external morphology of these half embryos differed from that of normal embryos, in that they possessed the complete structure of either the right or left side of an intact embryo, but not both, with the cut plane serving as the border. The left-half embryo lacked structures such as the right eye, balancer, gill and epidermis, whereas the right-half embryo lacked the structures seen on the left side alike (Fig. 2B-E, upper). However, the hearts formed in these half embryos were normal in external morphology. In the left-half embryos bisected at stage 11-25, rightward loops of the heart tubes were formed in $89-100 \%$ of cases (Fig. 2B, D, F). In the right-half embryos bisected at stage 11-18, leftward loops of the heart tubes were formed in $88-97 \%$ of specimens (Fig. 2C, F). These results were identical to those obtained in the ligation experiment on the two-cell embryos (Fig. 1); however, when embryos were bisected at stage 20-23, the direction of the heart-tube loop gradually shifted from leftward to rightward in the right-half embryos and became almost right (>88\% of cases) after stage 23 (Fig. 2E, F).

\section{Effects of the embryonic caudal region on looping of the heart tube}

We next examined the effects of the embryonic caudal region (which contains equivalent regions to the node of mammalian and avian embryos) on formation of the heart-tube loop. To disturb interactions between the left and right sides of the caudal region, the caudal one-third (1/3 of the posterior part) of the embryo was incised along the median sagittal plane (Fig. 3A). These experimented embryos had normal external morphology, except for a

Fig. 2. Hearts formed in bisected embryos. (A) Schematic representation of the bisection experiment. bp, blastopore; PCM, presumptive cardiac mesoderm; th, tungsten needle; $L$, left; $R$, right. (B-E) Right- and left-half embryos (stage 37) formed from an embryo bisected at the early gastrula (stage 11) (B,C) or late neurula stage (stage 21) (D,E). The rightand left-half embryos at stage 37 viewed from the dorsal side (upper pictures) and the heart tube viewed from the ventral side (lower pictures). Portions of the body wall and the pericardium were removed to expose the heart. bc, bulbus cordis; at, atrium; ve, ventricle. White arrow indicates the direction of the heart-tube loop. Scale bar: $0.5 \mathrm{~mm}$ (upper pictures), 0.1 $\mathrm{mm}$ (lower pictures). (F) Graphic representation of the relationship between the timing of bisection and the direction taken by each heart-tube loop formed in the bisected embryos. L-h, left-half embryo; $R$-h, right-half embryo. PCM is denoted in green in the illustration.

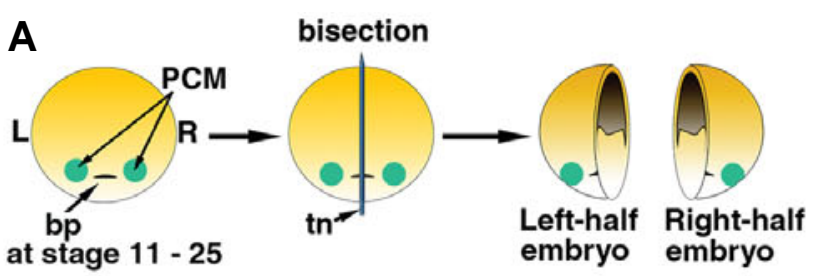

\section{at stage 11}

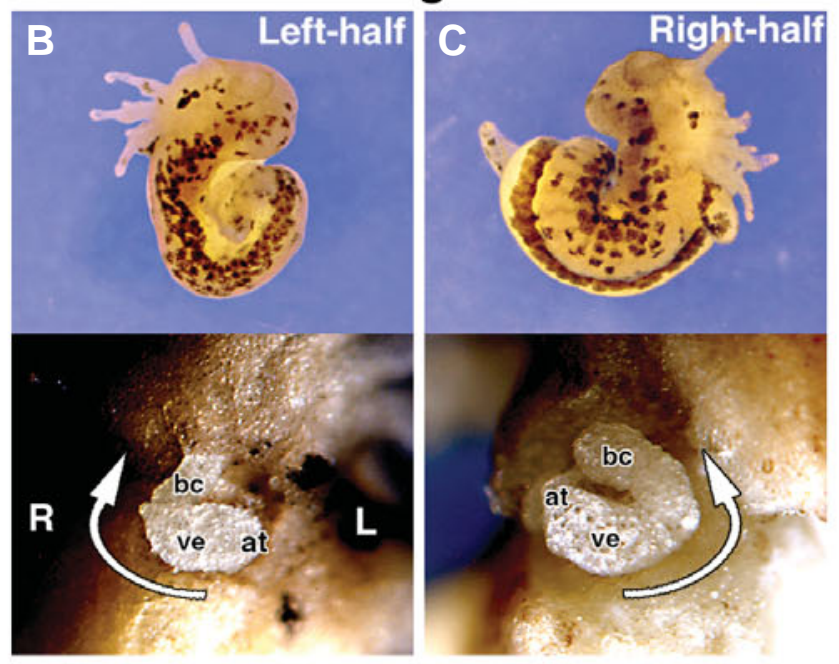

at stage 21
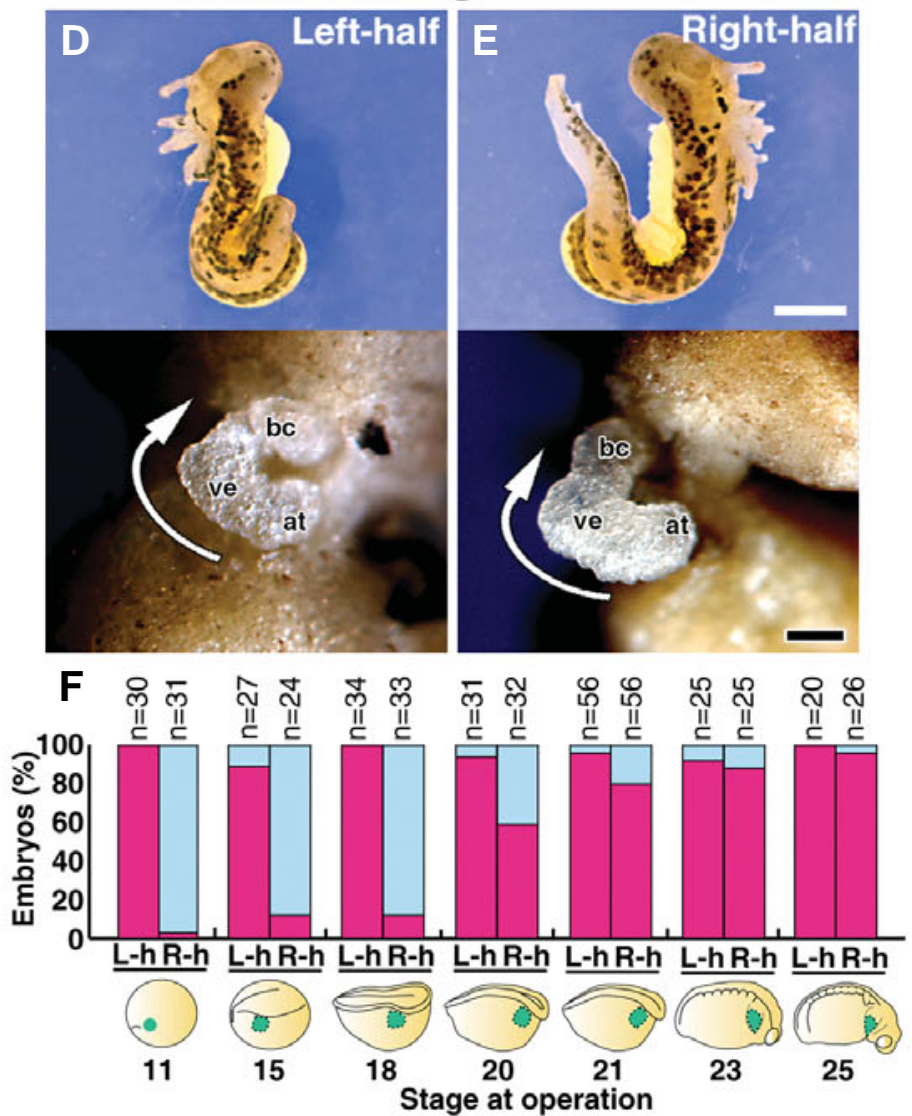

$\square$ Rightward looping $\square$ Leftward looping 
bifurcated tail at stage 36 (Fig. 3C, D, upper). It should be noted that the right and left PCM were fused in this experiment. In the embryos incised at stages 15 and 18, approximately one-half (55$63 \%$ ) of the loops were rightward while the remainders were leftward (Fig. 3B, C). This indicated that the embryos incised at these stages had lost the ability to form a normal rightward loop of heart tube, resulting in the loops twisting in random directions. If the caudal regions were incised at stage 20 , heart-tube loops showed the normal direction (rightward) in $92 \%$ of cases (Fig. 3B, D). These results supported the observation that heart-tube loops were directed rightward in the right-half embryos bisected after stage 20 (Fig. 2F). Together, the findings suggested that interactions in the caudal region from stage 15 to 18 are crucial for determining the direction of the heart-tube loop formed by PCM fusion, possibly the change in direction of the looping of heart tube derived from the right PCM.

We next resected the caudal one-third of right- and left-half embryos to block signals from the caudal region to the PCM and investigated the directions of heart-tube looping (Fig. 3E). In the resected embryos incubated until stage 36 , the external morphology was identical to that of the bisected embryos shown in Fig. 2, except for the absence of a tail (Fig. 3G-J, upper). The loops of the heart tubes were rightward in $>83 \%$ of tailless/left-half embryos operated on between stages 18 and 25, (Fig. 3F, G, I). In the tailless/right-half embryos operated by stage 23 , the heart-tube loops were leftward in $>83 \%$ of cases (Fig. 3F, H). However, the
A

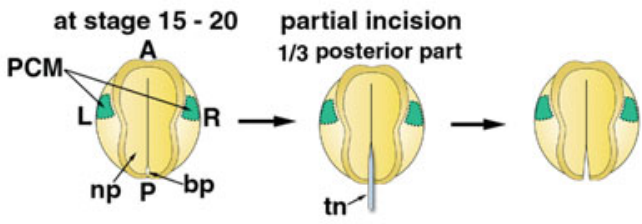

at stage 18

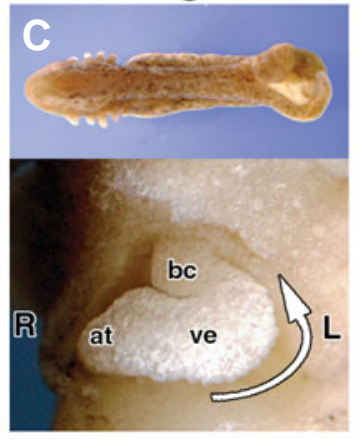

$\bar{E}$

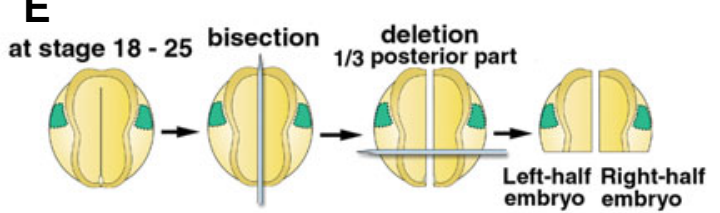

at stage 21
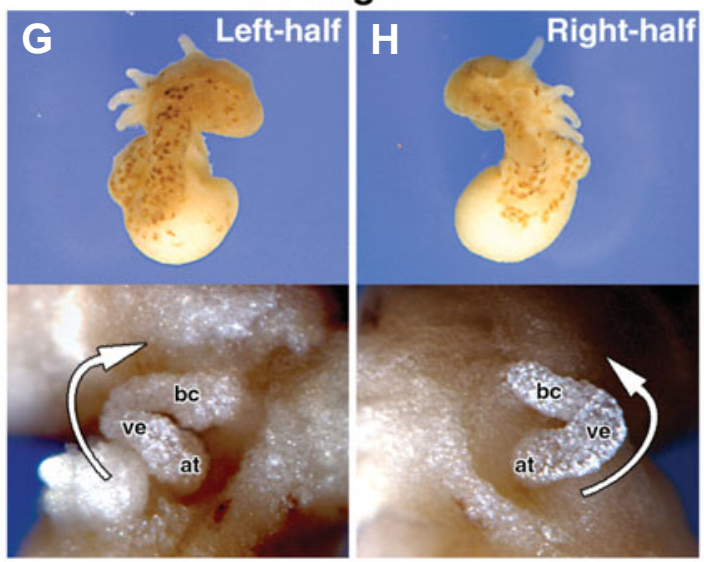

B
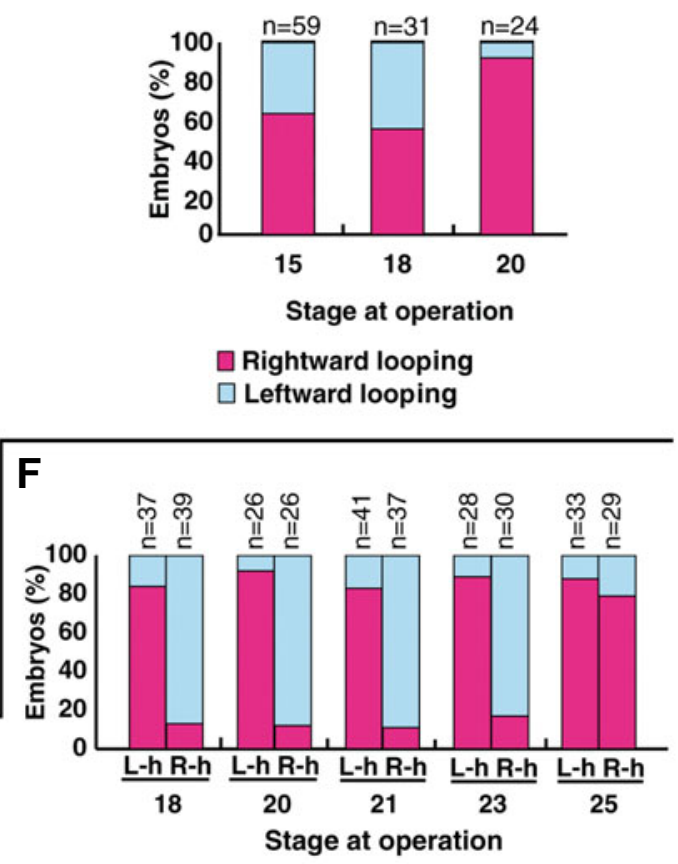

$\square$ Rightward looping $\square$ Leftward looping

at stage 25
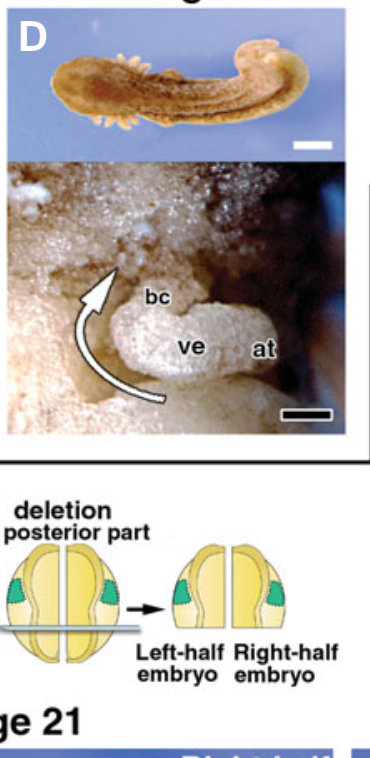
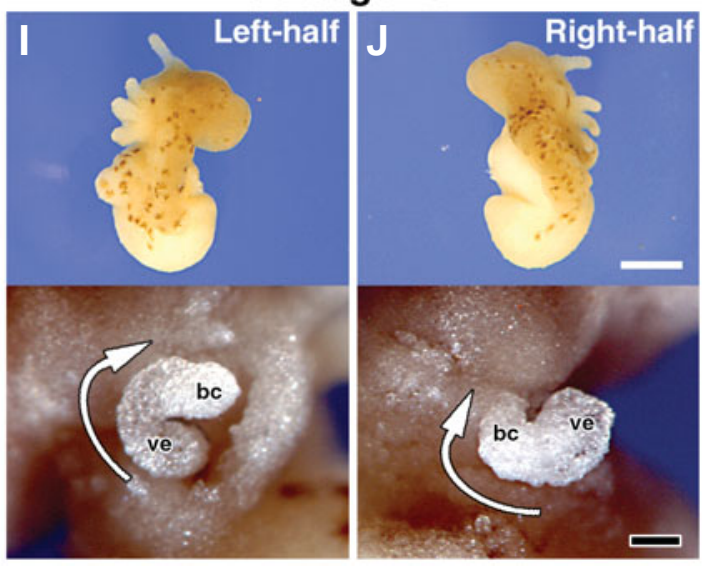

Fig. 3. Effects of the embryonic caudal region on looping of the heart tube. $(A-D)$ Incision of the caudal one-third $11 / 3$ of the posterior part) of the embryo. (A) Schematic representation of incising the caudal onethird. bp, blastopore; np, neural plate; $P C M$, presumptive cardiac mesoderm; tn, tungsten needle; $A$, anterior; $P$, posterior; $L$, left; $R$, right. (B) Graphic representation of the relationship between the timing of incision and the direction taken by each hearttube loop formed in the incised embryos. (C,D) Embryos at stage 36 developed from caudal incised embryos at stage 18 (C) or 20 (D), viewed from the dorsal side (upper pictures) with the heart tubes viewed from the ventral side (lower pictures). Portions of the body wall and the pericardium were removed to expose the heart. bc, bulbus cordis; at, atrium; ve, ventricle. White arrow indicates the direction of the loop of heart tube. Scale bar: $1 \mathrm{~mm}$ (upper pictures), $0.1 \mathrm{~mm}$ (lower pictures). (E-J) Half embryos with the caudal one-third region resected $11 / 3$ of the posterior part of the embryo). (E) Schematic representation of the bisection and resection of the caudal one-third of embryos. (F) Graphic representation of the relationship between the timing of operations and the direction taken by each heart-tube loop formed in the embryos. L-h, left-half embryo; $R$ - $h$, right-half embryo. (G-J) Embryos at stage 36 developed from embryos operated on at stage 21 (G, H) or 25 (I, J), viewed from the dorsal side (upper pictures) with the heart tubes viewed from the ventral side (lower pictures). The atrium is masked by the ventricle in the hearts (shown in I and J). Scale bar: $0.5 \mathrm{~mm}$ (upper pictures), $0.1 \mathrm{~mm}$ (lower pictures). 
direction of the loop was inverted rightward in $79 \%$ of the tailless/ right-half embryos operated on at stage 25 (Fig. 3F, J). These results indicate that the caudal region has an important role until stage 23 in the inversion of the looping direction in heart tube formed from the right PCM in right-half embryos.

\section{CyNodal expression in bisected embryos}

To investigate the relationship between the looping direction of the heart tube and the expression of CyNoda/in the lateral plate mesoderm, we performed whole-mount in situ hybridization on the right- and left-half embryos at stages 23-25. In the embryos bisected at stage 11, neither the right- nor the left-half embryos showed CyNodalexpression in the lateral plate mesoderm (Fig. 4A, B, F). Among the embryos bisected at stage 15, CyNodal expression was detected in $46 \%$ and $41 \%$ of the left- and right-half embryos, respectively (Fig. 4F). In the embryos bisected at stage 21, CyNoda/was expressed in all left-half embryos, but only in $38 \%$ of the right-half embryos (Fig. 4C, D, F). In intact embryos at stages 23-25, CyNodal expression localized in the left lateral plate mesoderm, but not in the right side of the body (Fig. 4E, F).

\section{Discussion}

Previous experimental findings including the ligation of twocell newt embryos (Spemann and Falkenberg, 1919) and the transplantation of PCMs into chick embryos (Hoyle et al., 1992) suggested that any intrinsic differences in loop formation between the right and left PCMs were derived from the right and left sides of an embryo, respectively. Halving experiments involving the ligation and bisection of early newt embryos in the present study strongly supported this possibility. We clearly demonstrated that heart tube derived from the right $\mathrm{PCM}$ looped toward the left, while heart tube derived from the left PCM looped toward the right. In our bisected embryos, right and left heart tubes were formed from right and left PCMs, respectively. The loops of these heart tubes were normally oriented rightward in left-half embryos irrespective of the developmental stage of the operation, but were reversed in right-half embryos bisected at stage 11-18. These results were identical to those obtained in the earlier ligation experiments on two-cell embryos, supporting that PCMs derived from the right and left sides of an embryo formed heart-tube loops with a mirror image orientation. However, during the later stages (18-21), the right-side PCM must receive a signal to alter the direction of the heart-tube looping from leftward to rightward, as both right and left PCMs possess the ability to form a rightward looping heart tube

Fig. 4. CyNodal expression in the separated right- and left-half embryos. (A, B) CyNodal expression was not detected in either the left(A) or right- (B) half embryos at stage 23 that developed from an embryo bisected at stage 11. (C, D) CyNodal expression (arrows) was observed, however, in those left- $(C)$ and right- (D) half embryos at stage 23 that developed from embryos bisected at stage 21. The images depict the left side of the left-half embryo $(A, C)$ and the right side of the right-half embryo (B,D). (E) CyNodal expression (arrow) on the left side of an intact normal embryo at stage 23. (F) Graphic representation of the percentage of CyNodal expression at stage 23-25 developed from bisected embryos. CyNodal expression was absent on the cut side (right side of the left-half embryos and left side of the right-half embryos) of all half embryos. A, anterior; $P$, posterior; $L-h$, left-half embryo; $R$ - $h$, right-half embryo; L-s, left side, $R$-s, right side. Scale bar: $0.5 \mathrm{~mm}$. prior to PCM fusion. Cytoskeletal organization (Latacha et al., 2005; Taber, 2006) and adhesion properties of heart tube cells (Garcia-Castro et al., 2000) play important roles in the looping of the developing heart tube and it seems likely that such factors cannot function properly unless both right and left PCMs can form a rightward loop at their fusion.

We also disturbed the interactions between right and left parts of the caudal area (equivalent to the nodal area of mammalian and avian embryos) to elucidate the involvement of this region in the inversion of heart-tube looping direction observed in the right-half embryos. In these experiments, loops of heart tubes were formed with random directions when the caudal region was separated by approximately stage 18 . This is probably because the perturbed interactions between the right and left caudal regions hampered inversion of the looping direction (from leftward to rightward) of heart tube formed from the right PCM. In mammalian and avian embryos, signal transduction within the nodal area, such as nodal flow by monocilia (Nonaka et al., 2002; McGrath et al., 2003; Tanaka etal., 2005) and $\mathrm{Ca}^{2+}$ signal transduction (McGrath et al., 2003; Raya et al., 2004), is important in determining the left-right asymmetry of various organs including the heart. The present results thus indicated that interactions between the right and left
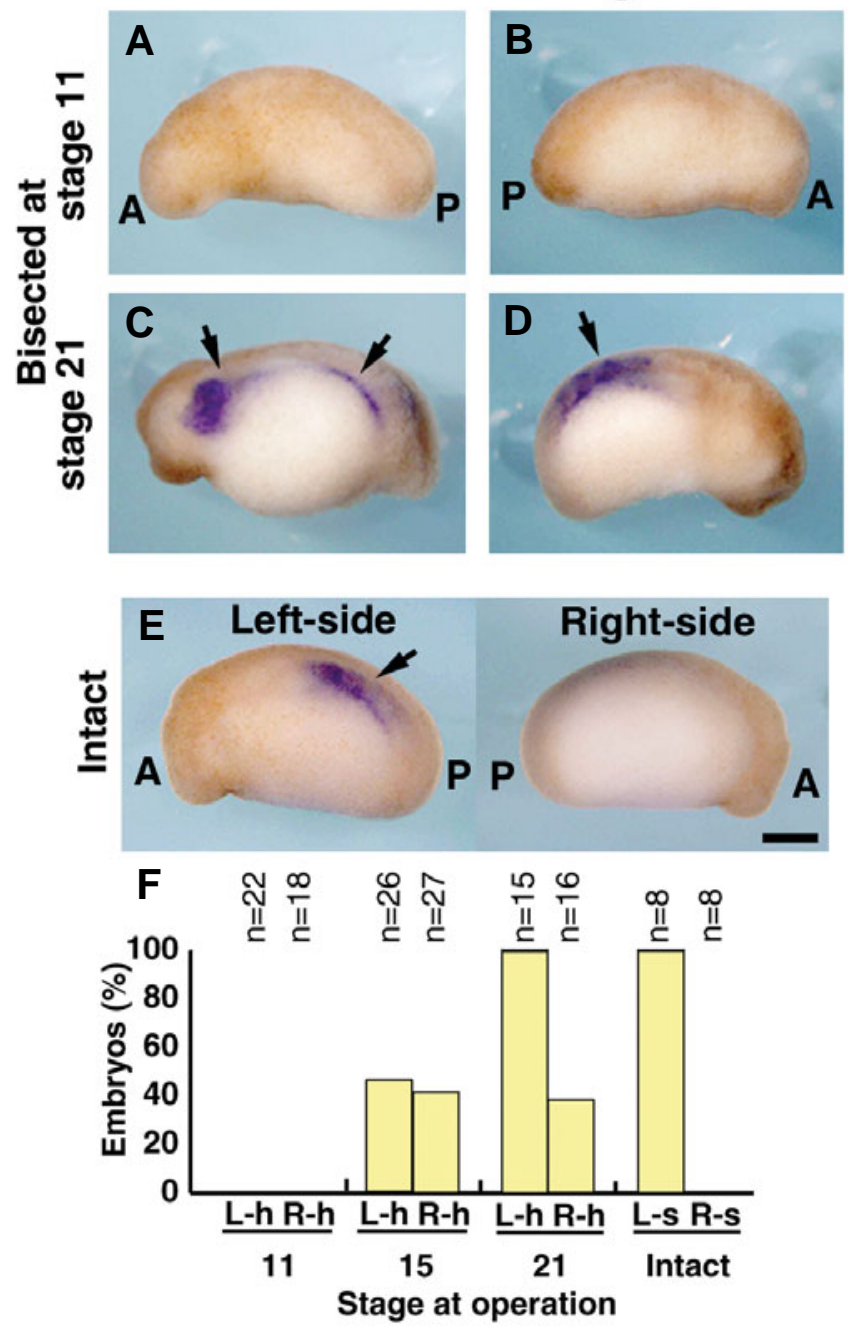

CyNodal expression at stage23 - 25 


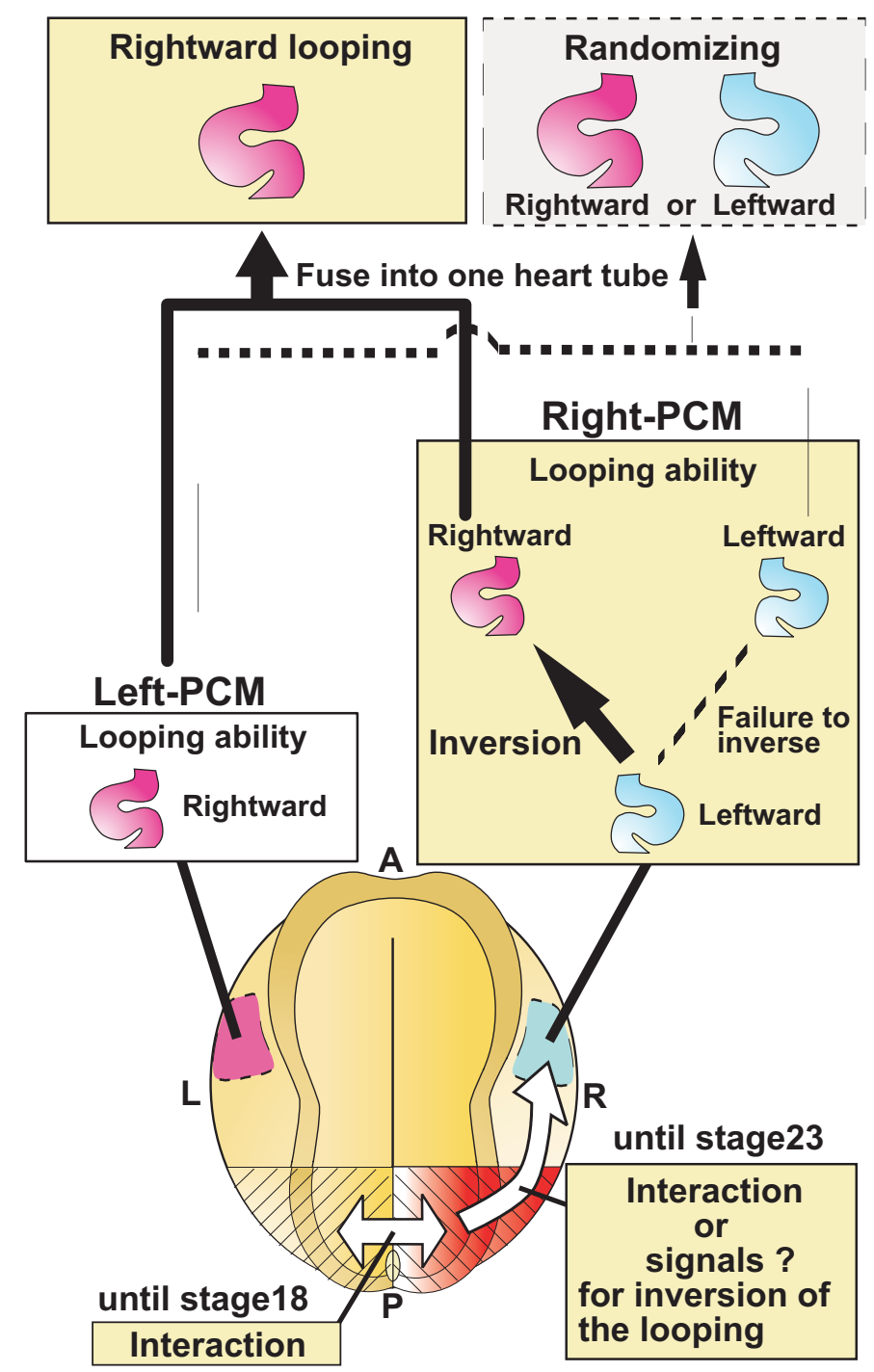

Fig. 5. Model of the proposed mechanism underlying formation of the rightward loop of the heart tube during newt cardiac development. During the early stages of embryonic development, the right presumptive cardiac mesoderm (PCM) can form a leftward loop of heart tube. This PCM, however, is altered in its potential to form a rightward loop of heart tube by the time that fusion of the right and left PCM occurs. By stage 18, interactions between the right and left sides in the embryonic caudal region (the area surrounded by the oblique lines) result in activation of the right caudal region. Between the activated right caudal region (highlighted in red) and the right PCM, interactions or unknown signals for inversion of the direction of looping are applied, resulting in the right PCM acquiring the ability to form a rightward (normal) loop of heart tube. In contrast, the left PCM retains the ability to form a rightward loop of heart tube during early and later stages of embryonic development. Fusion of the right and left PCM, both of which can form a rightward loop of heart tube, probably leads to formation of the heart with a rightward loop. If inversion of the direction of loop formation does not occur in the right PCM for any reason, the direction of the heart-tube loop formed after fusion of the right and left PCM will vary at random with the loop-forming potential between the right and left PCM. The heart illustration (ventral view) shows the pertinent characteristics of the PCM with regard to loop formation. PCM, presumptive cardiac mesoderm lactually located below the ectoderm). A, anterior; $P$, posterior; $L$, left; $R$, right. sides of the caudal region are important in determining the rightward looping of heart tube in amphibian embryos, as suggested for mammalian embryos (Nonaka et al., 2002; McGrath et al., 2003; Tanaka et al., 2005) and avian embryos (Raya et al., 2004).

The mechanism of determination of left-right asymmetry in vertebrates, various signals from the right and left sides of the node or its equivalent area to the anterior part of the embryo appears to involve induction of the expression of Nodal on the left side with concomitant suppression of Nodal expression on the right side (Levin et al., 1995; Collignon et al., 1996; Lowe et al., 1996; Lohr et al., 1997). This is followed by the restricted expression of transcription factors, such as Pitx2 on only the left side of the embryo (Campione et al., 1999; Patel et al., 1999; Campione et al., 2001; Yu et al., 2001; Mogi et al., 2003; Toyoizumi et al., 2005), BMP on the right side alone (Ramsdell et al., 1999; Branford et al., 2000; Monsoro-Burq and Le Douarin, 2000; Monsoro-Burq and Le Douarin, 2001) and SnR (Issac et al., 1997; Patel et al., 1999). It has been suggested that these factors are also important in determining the rightward direction of hearttube looping in mammalian, avian and amphibian embryos. Based on these previous reports, we evaluated the roles of the caudal region in this determination by resection in right- and lefthalf embryos. The caudal region was indispensable until around stage 23 for inverting the direction of the heart-tube loop in the separated right-half embryo, implicating the right caudal region, possibly signaling interactions exerted on the anterior right PCM until around stage 23 , in determining the direction of the looping of heart tube (Fig. 5).

We additionally examined the involvement of Nodal-related signaling in the heart-tube loop inversion in the separated righthalf embryo using bisection experiments. In normal embryos, CyNodal (a nodal-related gene in newts) expression was detected from stage 21 to 25 only on the left side of the embryo and was not seen on the right side (Ito etal., 2006). However, in stage23-25 embryos bisected at stage 15 or 21, CyNodal was expressed in $41 \%$ and $38 \%$ of the right-half embryos, respectively. Midline structures such as the notochord and the floor plate of neural tube suppress the expression of Nodal on the right sides of embryos (Danos and Yost, 1996; Lohr et al., 1997; Meno et al., 1998). To check for notochord formation in the bisected embryos, we incubated the right-half embryos (bisected at stage 21) until stage 36 when the midline structures are obvious and then prepared the specimens for histological observation. Notochord and a neural tube were clearly formed in $55 \%$ of the right-half embryos ( $n=20$, data not shown). Thus, the CyNodalexpression rate $(38 \%)$ in right-half embryos (bisected at stage 21 ) was approximately equal to the percentage of right-half embryos that did not form notochord $(45 \%)$. This result is similar to that reported by Lohr et al. (1997) that Xnr-1 (Xenopus nodal-related 1) expression was also seen in the right lateral plate mesoderm of embryos from which the midline structures (including the notochord) had been removed. Although CyNodal expression was noted in $41 \%$ and $38 \%$ of our right-half embryos separated at stages 15 and 21, respectively, inversion of the heart-tube loop direction was noted in $13 \%$ and $80 \%$ of the right-half embryos separated at stages 15 and 21, respectively. Furthermore, the CyNodal expression rates were $0 \%$ and $100 \%$ in the left-half embryos separated at stages 11 and 21 , respectively and nearly 
all of the loops of heart tube formed from these embryos were rightward (100\% and $96 \%$, respectively). Thus, CyNodalexpression in the half embryos was not correlated to the percentage of the looping direction of heart tube.

In conclusion, our study revealed that (1) the PCMs in the rightand left-half embryos independently formed heart-tube loops, (2) the looping directions of the heart tubes from the two halves were reverse when the embryos were separated at early stages and (3) the right PCM and the left PCM fused after both PCMs had gained the capacity to form heart-tube looping in the same direction (rightward looping). However, there are several questions that remain unanswered. How does each PCM loop in opposite directions (rightward looping of the left PCM, leftward looping of the right $\mathrm{PCM}$ ) in the early stages and how does the fusion of the left PCM looping to the rightward and the right $\mathrm{PCM}$ looping to the leftward result in a random looping direction. More extensive study will be needed in the future to address these important issues in cardiac development.

\section{Materials and Methods}

\section{Embryos}

In this study we used newt (Cynops pyrrhogaster) embryos, as fertilized newt eggs have large diameters $(2 \mathrm{~mm})$ allowing easy separation of the right and left halves. Embryos were obtained by injecting human chorionic gonadotropin (100 U) (Gestron, Denka Seiyaku Co., Kanagawa, Japan) into mature female newts twice, one day apart. The embryos were disinfected by immersion in $70 \%$ ethanol for 30 seconds and then washed gently with Steinberg's solution $(58 \mathrm{mM} \mathrm{NaCl}, 0.67 \mathrm{mM}$ $\mathrm{KCl}, \quad 0.34 \mathrm{mM} \mathrm{Ca}\left(\mathrm{NO}_{3}\right)_{2}, 0.83 \mathrm{mM} \mathrm{MgCl}{ }_{2}, 3 \mathrm{mM} \mathrm{N}-2-$ hydroxyethylpiperazine- $N$ 'ethansulfonic acid [HEPES] and $100 \mathrm{mg} / \mathrm{l}$ kanamycin sulfate, $\mathrm{pH}$ 7.2). The jelly capsule of each embryo was removed with fine forceps. The embryos were subsequently transferred into plastic dishes coated with $3 \%$ agarose and filled with Steinberg's solution. The fertilization membrane of each embryo was removed with fine forceps. The stage of embryonic development was rated in accordance with the criteria of Okada and Ichikawa (1947).

\section{Ligation experiment}

This experiment was carried out using a modification of the method reported by Spemann and Falkenberg (1919). We used two-cell embryos in which the first cleavage furrow passed the center of the grey crescent and had two blastomeres of the same size. Using a fine enamel-copper wire $(0.1 \mathrm{~mm}$ in diameter, Nilaco Corp., Tokyo, Japan), each two-cell embryo was ligated along the first cleavage furrow, together with the jelly capsule, into two separate blastomeres. The blastomere located left of the grey crescent was deemed the left-half embryo and that located to the right deemed the right-half embryo. The ligated embryo was incubated until stages $35-37$ at $20^{\circ} \mathrm{C}$ and the heart-tube loop direction was confirmed. To obtain accurate data, the following cases were excluded from the evaluation: embryos which failed to undergo equivalent separation, incomplete separations, morphologically incomplete embryos and embryos that failed to form a heart tube.

\section{Bisection, caudal incision and caudal resection}

Embryos were operated using fine tungsten needles under the following three sets of conditions. In experiment 1, embryos from the early gastrula to tail bud stages (stages 11-25) were bisected along the median sagittal plane and were separated into right- and left-half embryos. In experiment 2 , the caudal one-third ( $1 / 3$ of the posterior part) of embryos was incised along the median sagittal plane at the late gastrula to tailbud stages (stages 15-20). In experiment 3 , the embryos at the mid neurula to tail bud stages (stages 18-25) were bisected along the median sagittal plane, followed by resection of the caudal one-third ( $1 / 3$ of the posterior part) of each half embryo. The operated embryos were incubated at $20^{\circ} \mathrm{C}$ until stages 35-37 and the direction of the heart-tube loop was then confirmed. To obtain accurate data, morphologically incomplete embryos and embryos which failed to form a heart tube were excluded from the evaluations.

\section{Whole-mount in situ hybridization}

According to the method described elsewhere (Ito et al., 2006), bisected embryos that developed to stages 23-25 were subjected to whole-mount in situ hybridization, using an antisense RNA probe for CyNodal(Cynopus nodal-related gene). We recently showed that CyNodal mRNA expressed in the left lateral plate mesoderm at stages 21-25 (Ito et al., 2006).

\section{Acknowledgements}

This work was partly supported by grants from the International Cooperative Research Program (ICORP) of Japan Science and Technology Agency (JST) to M.A., the National Institute of Advanced Industrial Sciences and Technology (AIST) to M.A., the Life Science Foundation of Japan to K.T. and by a Grant-in-Aid for Exploratory Research from the Ministry of Education, Culture, Sports, Science and Technology of Japan (MEXT) to S.O.

\section{References}

BRANFORD, W.W., ESSNER, J.J. and YOST, H.J. (2000). Regulation of gut and heart left-right asymmetry by context-dependent interactions between Xenopus lefty and BMP4 signaling. Dev. Biol. 223: 291-306.

CAMPIONE, M., ROS, M.A., ICARDO, J.M., PIEDRA, E., CHRISTOFFELS, V.M., SCHWEICKERT, A., BLUM, M., FRANCO, D. and MOORMAN, A.F. (2001). Pitx2 expression defines a left cardiac lineage of cells: evidence for atrial and ventricular molecular isomerism in the iv/iv mice. Dev. Biol. 231: 252-264.

CAMPIONE, M., STEINBEISSER, H., SCHWEICKERT, A., DEISSLER, K., VAN BEBBER, F., LOWE, L.A., NOWOTSCHIN, S., VIEBAHN, C., HAFFTER, P., KUEHN, M.R. and BLUM, M. (1999). The homeobox gene Pitx2. mediator of asymmetric left-right signaling in vertebrate heart and gut looping. Development 126: 1225-1234.

COLLIGNON, J., VARLET, I. and ROBERTSON, E. (1996). Relationship between asymmetric nodal expression and the direction of embryonic turning. Nature 381: 155-158.

DANOS, M. and YOST, H. (1996). Role of notochord in specification of cardiac leftright orientation in zebrafish and Xenopus. Dev. Biol. 177: 96-103.

GARCÍA-CASTRO, M. I., VIELMETTER, E. and BRONNER-FRASER, M. (2000). $\mathrm{N}$-Cadherin, a cell adhesion molecule involved in establishment of embryonic left-right asymmetry. Science 288: 1047-1051.

HOYLE, C., BROWN, N. A. and WOLPERT, L. (1992). Development of left/right handedness in the chick heart. Development 115: 1071-1078.

ISAAC, A., SARGENT, M.S. and COOKE, J. (1997). Control of vertebrate left-right asymmetry by a snail-related zinc finger gene. Science 275: 1301-1304.

ITO, Y., OINUMA, T., TAKANO, K., KOMOZAKI, S., OBATA, S. and ASASHIMA, M. (2006). CyNodal, the Japanese newt noda-related gene, is expressed in the left side of the lateral plate mesoderm and diencephalon. Gene Expr. Patterns. 6: 294-298.

LATACHA, K.S., RÉMOND, M.C., RAMASUBRAMANIAN, A., CHEN, A.Y., ELSON, E.L. and TABER, L.A. (2005). Role of actin polymerization in bending of the early heart tube. Dev. Dyn. 233: 1272-1286.

LEVIN, M. (2005). Left-right asymmetry in embryonic development: a comprehensive review. Mech. Dev. 122: 3-25.

LEVIN, M., JOHNSON, R., STERN, C., KUEHN, M. and TABIN, C. (1995). A molecular pathway determining left-right asymmetry in chick embryogenesis. Cel/82: 803-814.

LOHR, J., DANOS, M. and YOST, H. (1997). Left-right asymmetry of a nodal related gene is regulated by dorsoanterior midline structures during Xenopus development. Development 124: 1465-1472. 


\section{K. Takano et al.}

LOWE, L.A., SUPP, D.M., SAMPATH, K., YOKOYAMA, T., WRIGHT, C.V., POTTER, S.S., OVERBEEK, P. and KUEHN, M.R. (1996). Conserved left-right asymmetry of noda/expression and alterations in murine situs inversus. Nature 381: 158-161.

MÄNNER, J. (2000). Cardiac looping in the chick embryo: a morphological review with special reference to terminological and biomechanical aspects of the looping process. Anat. Rec. 259: 248-262.

MCGRATH, J., SOMLO, S., MAKOVA, S., TIAN, X. and BRUECKNER, M. (2003). Two populations of node monocilia initiate left-right asymmetry in the mouse. Cel/114: 61-73.

MENO, C., SHIMONO, A., SAIJOH, Y., YASHIRO, K., MOCHIDA, K., OHISHI, S. NOJI, S., KONDOH, H. and HAMADA, H. (1998). Lefty-1is required for left-right determination as a regulator of lefty-2 and nodal. Cel/94: 287-297.

MOGI, K., GOTO, M., OHNO, E., AZUMI, Y., TAKEUCHI, S. and TOYOIZUMI, R. (2003). Xenopus neurula left-right asymmetry is respecified by microinjecting TGF-beta5 protein. Int. J. Dev. Biol. 47: 15-29.

MONSORO-BURQ, A.H. and LE DOUARIN, N. (2000). Left-right asymmetry in BMP4 signalling pathway during chick gastrulation. Mech. Dev. 97: 105-108.

MONSORO-BURQ, A.H. and LE DOUARIN, N. (2001). BMP4 plays a key role in left-right patterning in chick embryos by maintaining sonic hedgehog asymmetry. Mol. Cel/7: 789-799.

NONAKA, S., SHIRATORI, H., SAIJOH, Y. and HAMADA, H. (2002). Determination of left-right patterning of the mouse embryo by artificial nodal flow. Nature 418 : 96-99.

OKADA, Y. and ICHIKAWA, M. (1947). Normal table of Triturus pyrrhogaster. Jpn. J. Exp. Morphol. 3: 1-6.

PATEL, K., ISAAC, A. and COOKE, J. (1999). Noda/signalling and the roles of the transcription factors SnRand Pitx2in vertebrate left-right asymmetry. Curr. Biol. 9: 609-612.

RAMSDELL, A.F. (2005). Left-right asymmetry and congenital cardiac defects:
Getting to the heart of the matter in vertebrate left-right axis determination. Dev. Biol. 288: 1-20.

RAMSDELL, A.F. and YOST, H.J. (1999). Cardiac looping and the left- right axis: antagonism of left-sided Vg1 activity by a right-sided ALK2-dependent BMP pathway. Development 126: 5195-5205.

RAYA, A., KAWAKAMI, Y., RODRIGUEZ-ESTEBAN, C., IBANES, M., RASSKINGUTMAN, D., RODRIGUEZ-LEON, J., BUSCHER, D., FEIJO, J.A. and IZPISUA BELMONTE, J.C. (2004). Notch activity acts as a sensor for extracellular calcium during vertebrate left-right determination. Nature 427: 121-128.

SPEMANN, H. and FALKENBERG, H. (1919). Uber asymmetrische Entwicklung und Situs inversus viscerum bei Zwillingen und Doppelbildungen. Arch. Entwick. Org. 45: 371-422.

TABER, L. A. (2006). Biophysical mechanisms of cardiac looping. Int. J. Dev. Biol. 50: 323-332.

TANAKA, Y., OKADA, Y. and HIROKAWA, N. (2005). FGF-induced vesicular release of Sonic hedgehog and retinoic acid in leftward nodal flow is critical for left-right determination. Nature 435: 172-177.

TOYOIZUMI, R., OGASAWARA, T., TAKEUCHI, S. and MOGI, K. (2005). Xenopus nodal related-1 is indispensable only for left-right axis determination. Int. J. Dev. Biol. 49: 923-938.

YU, X., ST AMAND, T.R., WANG, S., LI, G., ZHANG, Y., HU, Y.P., NGUYEN, L., QIU, M.S. and CHEN, Y.P. (2001). Differential expression and functional analysis of Pitx2 isoforms in regulation of heart looping in the chick. Development 128: 1005-1013.

Received: 4th November 2006 Reviewed by Referees: 1st December 2006 Modified by Authors and Accepted for Publication: 10th January 2007 Published Online: 18th May 2007 Edited by: Makoto Asashima 


\section{The Spemann-Mangold}

\section{Organizer}

Edited by Eddy M. De Robertis and Juan A réchaga

Preface

by E.M. DeR obertis and J. A réchaga

HERITAGE OF THE 1924 ARTICLE BY HANS SPEMANN AND HILDE MANGOLD

Introducing the Spemann-Mangold organizer: experiments and insights that generated a

key concept in developmental biology

by $K$. Sander and P. Faessler

Induction of embryonic primordia by implantation of organizers from a different species

Reprint of the original 1924 article by $\mathrm{H}$ ans Spemann and $\mathrm{H}$ ilde $\mathrm{M}$ angold, translated into

English by Viktor $\mathrm{H}$ amburger

Developmental biology of amphibians after $\mathrm{H}$ ans Spemann in Germany

by H. Grunz

Spemann's heritage in Finnish developmental biology by L. Saxén

Spemann's influence on Japanese developmental biology

by M. A sashima and T. Okada

Contribution of the Belgian school of embryology to the concept of neural induction by the organizer

by $\mathrm{H}$. Alexandre

Contrasting influences of the organizer and induction concepts on the scientific activity of French embryologists

by J.-C. Beetschen and A.-M. Duprat

Consequences of the Spemann-Mangold organizer concept for embryological research in Russia: personal impressions

by A.T. M ikhailov and N.A. Gorgolyuk

The organizer concept and modern embryology: Anglo-American perspectives by $T$. $H$ order

THE ORGANIZER CONCEPT: OVERVIEWS AND THEORETICAL APPROACHES

Evolution of the organizer and the chordate body plan by J. Gerhart

Continuity and change: paradigm shifts in neural induction by S. Gilbert

Formation and maintenance of the organizer among vertebrates by $K$. Joubin and C.D. Stern

O rganizer and axes formation as a self-organizing process by H. M einhardt

ORGANIZER RESEARCH TODAY

Molecular mechanisms of cell-cell signalling by the Spemann-Mangold organizer by E.M. De Robertis, O. Wessely, M. Oelgeschläger, B. Brizuela, E. Pera, J. Larraín, J. A breu and D. Bachiller

Formation of a functional morphogen gradient by a passive process in tissue from the early Xenopus embryo

by N. M CD owell, J.B. Gurdon and D.J. Grainger

A study of Xlim1 function in the Spemann-Mangold organizer

by L. Kodjabachian, A.A. Karavanov, H. Hikasa, N.A. Hukriede, T. A oki, M. T aira and I.B. Dawid

Making mesoderm - upstream and downstream of Xbra by J. Smith

Regulation of convergent extension in Xenopus by Wnt5a and Frizzled-8 is independent of the canonical Wnt pathway

by J.B. Wallingford, K.M. Vogeli and R.M. H arland

Generation of the germ layers along the animal-vegetal axis in Xenopus laevis by H. Yasuo and P. Lemaire

Dickkopf 1 and the Spemann-Mangold head organizer by C. Niehrs, O. Kazansakaya, W.Wu, and A. Glinka

Siamois cooperates with TGF $\beta$ signals to induce the complete function of the SpemannMangold organizer

by M.J. Engleka and D. S. Kessler

\section{ORDER FORM}

I would like to order cop(y/ies) of the Int. J. Dev. Biol. Special Issue “The Spemann-Mangold Organizer” (Vol. 45, N 1)

at US\$ 70 or Euro $€ \overline{70}$ per copy (including post and packaging). Total to be charged: US\$ / Euro $€$ (please specify currency)

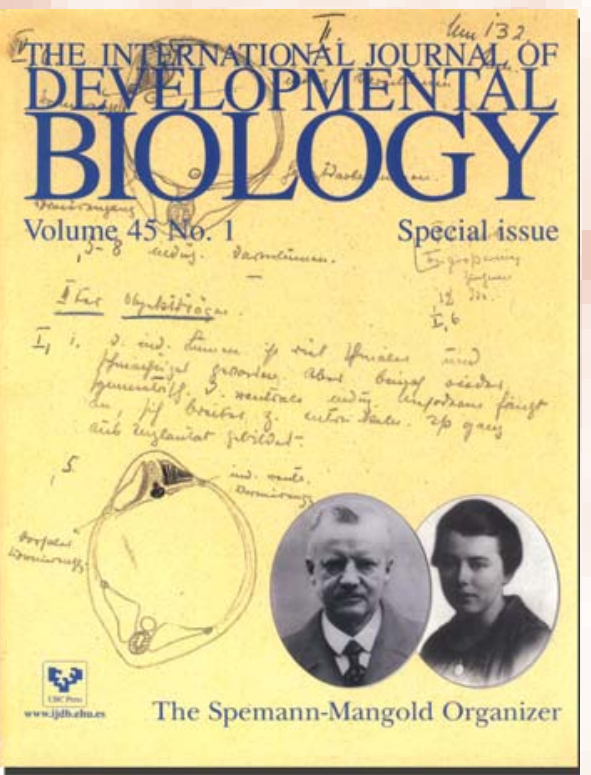

The Spemann-Mangold organizer: the control of fate specification and morphogenetic rearrangements during gastrulation in Xenopus by T. B ouwmeester

Functional analysis of the Xenopus frizzled 7 protein domains using chimeric receptors by R.K. Swain, A. M edina and H. Steinbeisser

Fox (forkhead) genes are involved in the dorso-ventral patterning of the Xenopus mesoderm

by H. El-H odiri, N. Bhatia-Dey, K. Ken yon, K. Ault, M. Dirksen and M. Jamrich

In vitro induction systems for analyses of amphibian organogenesis and body patterning by T. Ariizumi and M. Asashima

The avian organizer

by T. B oettger, H. Knoetgen, L. Wittler and M. Kessel

Nodal signaling and the zebrafish organizer by A.F. Schier and W.S. T albot

The role of the homeodomain protein Bozozok in Zebrafish axis formation by $L$. Solnica-K rezel and W. Driever

Role of the anterior visceral endoderm in restricting posterior signals in the mouse embryo

by A. Perea-Gomez, M. Rhinn and S. -L. Ang

Roles of Sox factors in neural determination: conserved signaling in evolution? by Y. Sasai

G etting your head around $H$ ex and $H$ esxl : forebrain formation in mouse by J.P. M artínez Barbera, R.S.P. Beddington

The role of 0 tx 2 in organizing the anterior patterning in mouse by A. Simeone and D. A campora

Defects of the body plan of mutant embryos lacking Lim1, 0 tx2 or $\mathrm{Hnf3} \beta$ activity by S.J. Kinder, T.E. T sang, S.-L. Ang, R.R. Behringer and P.P.L. T am

0 tx2 and $\mathrm{Hnf} \beta$ genetically interact in anterior patterning by 0 . Jin, K. H arpal, S.L. Ang and J. Rossant

The isthmic organizer and brain regionalization by S. M artínez

Early neurogenesis in amniote vertebrates by N. Le Douarin

\section{ORDER BY}

Web: http://www.intjdevbiol.com

E-mail: ijdb@ehu.es (include the information indicated above)

FAX: $\quad+34-94-601-3266$

FAX: $+34-94-601-3266$

The International Journal of Developmental Biology

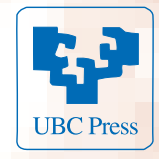

UBC Press - Editorial Service University of the Basque Country E-48940 Leioa, (Vizcaya)

SPAIN 